\title{
Microbiomes associated with bovine periodontitis and oral health
}

\author{
Ana C. Borsanelli ${ }^{\mathrm{a}}$, David F. Lappin ${ }^{\mathrm{b}}$, Lorenzo Viora ${ }^{\mathrm{c}}$, David Bennett ${ }^{\mathrm{c}}$, Iveraldo S. Dutra ${ }^{\mathrm{a}}$, \\ Bernd W. Brandt ${ }^{\mathrm{d}}$, Marcello P. Riggio ${ }^{\mathrm{b}, *}$ \\ a São Paulo State University (Unesp), School of Veterinary Medicine, Araçatuba Campus, São Paulo, Brazil \\ ${ }^{\mathrm{b}}$ Dental School, University of Glasgow, Glasgow, UK \\ ' School of Veterinary Medicine, University of Glasgow, Glasgow, UK \\ d Department of Preventive Dentistry, Academic Centre for Dentistry Amsterdam, University of Amsterdam and VU University Amsterdam, Amsterdam, Netherlands
}

\section{A R T I C L E I N F O}

\section{Keywords:}

Periodontal disease

Cattle

High-throughput sequencing

Microbiome

Bacteria

\begin{abstract}
A B S T R A C T
Periodontitis is an infectious polymicrobial, immuno-inflammatory disease of multifactorial aetiology that has an impact on the health, production and welfare of ruminants. The objective of the present study was to determine the microbial profiles present in the gingival sulcus of cattle considered periodontally healthy and in the periodontal pocket of animals with periodontitis lesions using high-throughput bacterial 16S rRNA gene sequencing. Subgingival biofilm samples were collected from 40 cattle with periodontitis and 38 periodontally healthy animals. In total, 1923 OTUs were identified and classified into 395 genera or higher taxa. Microbial profiles in health differed significantly from periodontitis in their composition $(p<0.0001, \mathrm{~F}=5.30$; PERMANOVA) but no statistically significant differences were observed in the diversity of healthy and periodontitis microbiomes. The most prevalent taxa in health were Pseudomonas, Burkholderia and Actinobacteria, whereas in disease these were Prevotella, Fusobacterium and Porphyromonas. The most discriminative taxa in health were Gastranaerophilales, Planifilum and Burkholderia, and in disease these were Elusimicrobia, Synergistes and Propionivibrio. In conclusion, statistically significant difference exists between the microbiome in bovine oral health and periodontitis, with populations showing $72.6 \%$ dissimilarity. The diversity of the bacteria found in health and periodontitis were similar and bacteria recognised as periodontal pathogens showed increased abundance in disease. In this context, the main components of bacterial homeostasis in the biofilm of healthy sites and of dysbiosis in periodontal lesions provide unprecedented indicators for the evolution of knowledge about bovine periodontitis.
\end{abstract}

\section{Introduction}

Periodontitis is a polymicrobial infectious disease initiated by a synergistic and dysbiotic microbial community (Hajishengallis and Lamont, 2012) that affects the health, production and welfare of ruminants. Usually neglected in animal production, it is a purulent, chronic and progressive infectious process that causes cumulative changes that occur throughout the lives of animals that is characterised by periodontal pocket formation, gingival recession, mobility, loss of clinical insertion and premature tooth loss (Page and Schroeder, 1976; Döbereiner et al., 2000; Borsanelli et al., 2016a).

The natural occurrence of periodontal lesions in sheep and cattle has been recorded in several countries and epidemiological contexts (Aitchison and Spence, 1984; Döbereiner et al., 2000; Ingham, 2001; Fadden et al., 2015; Borsanelli et al., 2016a).

Some species of oral bacteria, such as Porphyromonas gingivalis and
Aggregatibacter actinomycetemcomitans, have been considered to be important in the development of periodontal disease in humans and other animal species. In cattle, the participation of some potential periodontopathogens in lesions of the disease has also been recognised, including Fusobacterium nucleatum, Trueperella pyogenes and some species of the Porphyromonas, Prevotella and Treponema genera (Blobel et al., 1987; Dutra et al., 2000; Borsanelli et al., 2015a, 2015b).

An important step for understanding the participation of putative bacterial pathogens in periodontitis is to determine the bacterial composition in the healthy gingival sulcus and in the periodontal pocket. It has been estimated that approximately $50 \%$ of the human oral microbiota is uncultivable (Socransky et al., 1963), and an analogous situation is likely in the bovine oral cavity.

At present, it is possible to determine almost all the community of commensal and potentially pathogenic bacteria that inhabit the bovine oral cavity, both in health and in periodontitis, using culture-

\footnotetext{
* Corresponding author at: Oral Sciences Research Group, Level 9, Glasgow Dental Hospital \& School, 378 Sauchiehall Street, Glasgow G2 3JZ, UK.

E-mail address: Marcello.Riggio@glasgow.ac.uk (M.P. Riggio).
} 
independent methods. Bacterial 16S rRNA gene sequencing allows detection of not only cultivable species but also uncultivable bacteria and novel species that may be important in disease pathogenesis. This method has already been used to determine the oral bacterial community of horses, sheep and dogs with and without periodontal lesions (Riggio et al., 2011, 2013; Kennedy et al., 2016) and to determine the oral microbiome of periodontally healthy dogs and cats (Dewhirst et al., 2012; Holcombe et al., 2014; Sturgeon et al., 2014).

The objective of the present study was to determine the microbial profiles present in the gingival sulcus of cattle considered periodontally healthy and in the periodontal pockets of animals with periodontitis lesions using high-throughput bacterial 16S rRNA gene sequencing.

\section{Materials and methods}

\subsection{Collection of dental plaque}

Two-hundred dental arches of bovines were examined at a local slaughterhouse in Scotland during the period September to November 2015 and dental plaque samples were collected. Since periodontitis includes inflammatory alterations of the gingival tissue and a progressive loss of periodontal attachment and alveolar bone, the criteria for the diagnosis of the disease was the presence of gingival retraction (i.e. the tooth root was visible at the gingival margin), the existence of a periodontal pocket (the distance from the gingival margin to the bottom of the periodontal pocket as measured with a graduated universal periodontal probe) greater than $5 \mathrm{~mm}$ in depth and suppuration (presence of pus inside the periodontal pocket; usually observed when curetting the bottom of the pocket). Since samples were collected postmortem it was not possible to evaluate bleeding on probing. The periodontally healthy group had no evidence of gingival retraction, no periodontal pockets, no suppuration and no evidence of any other oral disease. The probe was inserted to the base of the periodontal pocket, applying a light force and moved gently around the tooth surface and pocket depth measurement obtained. Samples were collected within 30 min of death.

Subgingival plaque was collected from the periodontal pocket of 40 cattle with periodontitis and from the gingival margin around premolar 2-premolar 3 of 38 periodontally healthy cattle with the aid of a sterile curette. All samples were placed in $250 \mu \mathrm{L}$ of RNAlater (Sigma-Aldrich, Gillingham, UK) and stored at $-20^{\circ} \mathrm{C}$ until required.

\subsection{DNA preparation}

Subgingival plaque samples were mixed by vortexing for $30 \mathrm{~s}$. To $150 \mu \mathrm{L}$ of each sample was added $200 \mu \mathrm{L}$ phenol saturated with Tris- $\mathrm{HCl}$ ( $\mathrm{pH}$ 8.0), $200 \mu \mathrm{L}$ lysis buffer and $250 \mu \mathrm{L}$ glass beads $(0.1 \mathrm{~mm}$ ) suspended in TE buffer. Bead beating was conducted in a BioSpec MiniBeadbeater for $2 \mathrm{~min}$ at 2100 oscillations/min. DNA was then purified using the AGOWA mag Mini DNA Isolation Kit (AGOWA, Berlin, Germany).

\subsection{High-throughput sequencing}

Bacterial 16S rRNA genes were amplified using primers GTGCCAG CMGCCGCGGTAA (forward) and GGACTACHVGGGTWTCTAAT (reverse) that target the V4 region. Amplicon libraries were purified, analysed and paired-end $(2 \times 251 \mathrm{bp})$ sequenced using the Illumina MiSeq as described previously (Kennedy et al., 2016).

\subsection{Bioinformatics analysis}

USEARCH version 8.0.1623 (Edgar and Flyvbjerg, 2015) was used to merge, process and cluster sequencing reads. Following merging, quality filtering (maximum expected error rate 0.5 and no ambiguous bases allowed) was conducted and sequences clustered into operational taxonomic units (OTUs) using the settings: uparse_maxdball 1200, only de novo chimera checking, usearch_global with -maxaccepts 8 -maxrejects 64 -maxhits 1 . The most abundant sequence of each OTU was selected using QIIME version 1.8.0 (Caporaso et al., 2010) and a taxonomy was then assigned with the RDP classifier (Cole et al., 2009) with a minimum confidence of 0.8 and the $97 \%$ representative sequence set based on the SILVA rRNA database, release 119 for QIIME (Quast et al., 2013).

\subsection{Statistical analysis}

Normalisation of sequencing depth was achieved by random subsampling of the dataset to $50 \%$. Diversity analysis (Shannon Diversity Index, Chao-1 estimate of total species richness), data ordination by principal component analysis (PCA) and assessment of differences between microbial profiles of the two groups by one-way PERMANOVA were performed using PAleontological STatistics (PAST; v3.02) software (Hammer et al., 2001). PERMANOVA was used with Bray-Curtis similarity distance. For PCA, the OTU dataset was additionally normalised by $\log _{2}$-transformation. Diversity output was compared using the Mann-Whitney U test in SPSS (version 21.0). Linear discriminant analysis effect size (LEfSe) was used to determine which OTUs and taxa contribute to differences between the groups (Segata et al., 2011).

\section{Results}

\subsection{Sequencing output}

Sequencing generated 1,296,437 read pairs and after merging and quality filtering $86.5 \%$ of these (i.e. 1,122,045) remained. Following clustering (including chimera checking) $88.5 \%(992,913)$ of these $1,122,045$ sequences were mapped to OTUs and were thus present in the OTU table used for downstream analysis. After random subsampling at $50 \%, 1923$ OTUs were identified and classified into 395 genera or higher taxa. The most prevalent genera or higher taxa are shown in Fig. 1.

\subsection{Microbial profile analysis}

Differences between the bovine oral microbiomes of oral health and periodontitis were evident as determined by principal component analysis (Fig. 2). Generally, the healthy and periodontitis samples tended to cluster separately and the healthy samples demonstrated lower intra-sample variability relative to the periodontitis samples. A statistically significant difference between the microbial profiles of health and disease was observed ( $p<0.001, \mathrm{~F}=5.30$, PERMANOVA). Bray-Curtis analysis demonstrated $72.6 \%$ dissimilarity between the two groups. No statistically significant differences were observed in species richness or diversity of healthy and periodontitis microbiomes (Fig. 3).

On average, healthy samples contained 238 OTUs (SD 158, range 66-698), while the periodontitis samples contained 245 OTUs (SD 114, range 79-577).

\subsection{Differences in composition between healthy and periodontitis samples}

From 395 genera or higher taxa, 45 taxa were statistically significantly different between the two groups ( $p<0.05$ ); of these, 25 taxa had a linear discriminant analysis (LDA) score above 2 and the majority (17 of 25 taxa) were associated with disease (Fig. 4). Taxa are ranked by the effect size in LEfSe.

The most discriminative taxa in the samples of healthy animals were Gastranaerophilales, Planifilum, Burkholderia and Arcobacter; in animals with periodontitis, the most discriminative taxa were Elusimicrobia, Synergistes, Propionivibrio and Fusobacteria (Fig. 4). 


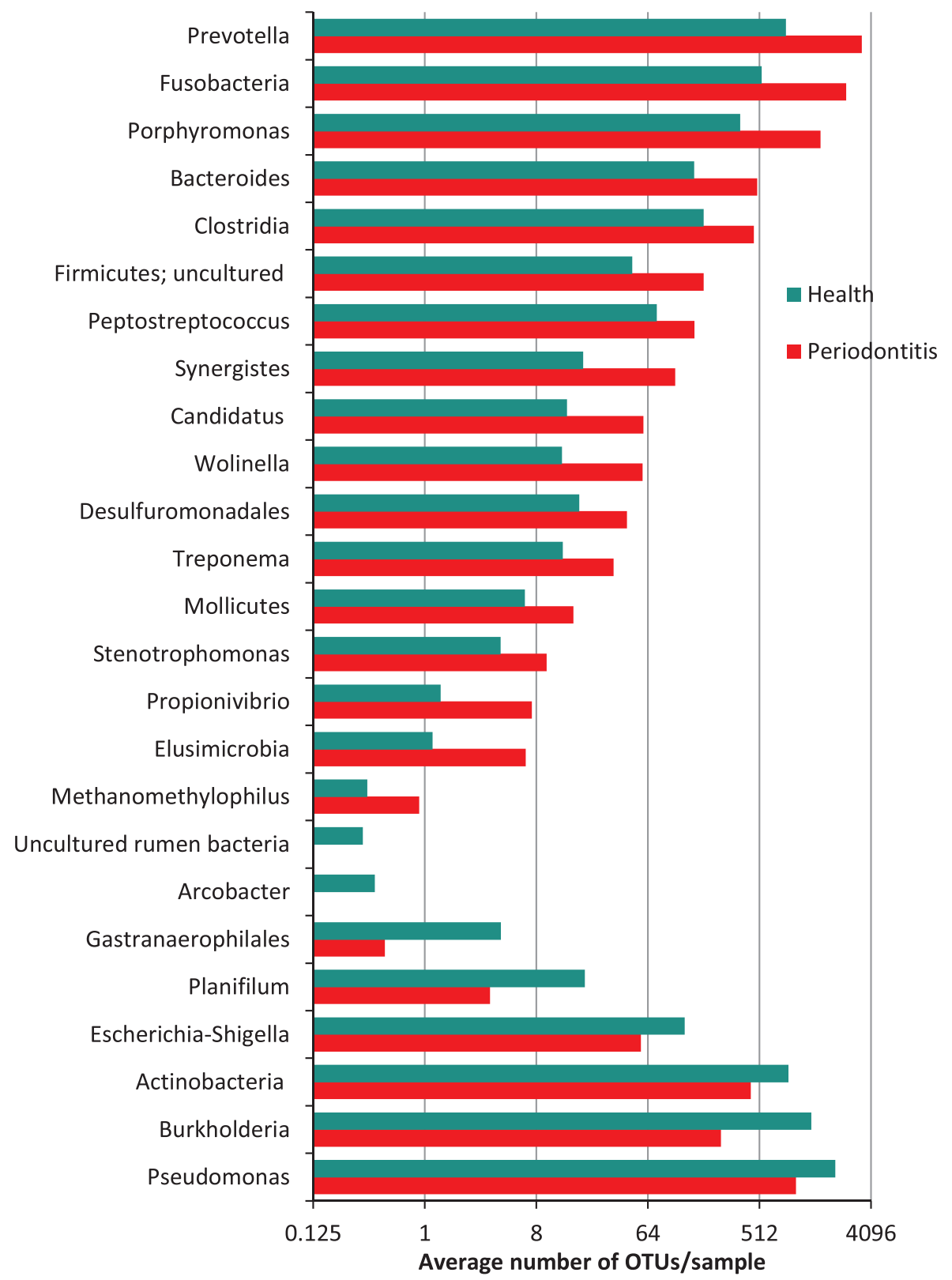

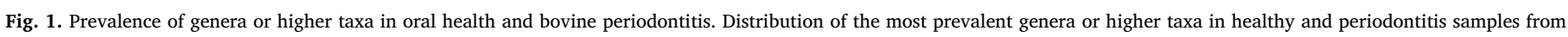

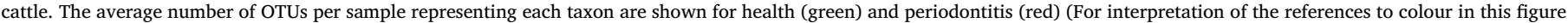
legend, the reader is referred to the web version of this article).

\section{Discussion}

The present study is the first to use high-throughput 16S rRNA gene sequencing to compare bacterial populations present in bovine oral health and periodontitis. It was shown that a statistically significant difference exists between the microbiome in bovine oral health and periodontitis, with populations showing $72.6 \%$ dissimilarity. This represents a considerable advance in knowledge over what was previously documented for the oral microbial communities of cattle.

The human and animal oral cavity houses a complex and diverse microbial community that plays a critical role in health and disease. To date, approximately 700 species have been described in the human oral cavity, of which approximately $32 \%$ have not yet been cultivated (Chen et al., 2010). Recent advances in gene sequencing and bioinformatics technology have enabled the taxonomic identification of previously unknown microorganisms and made it possible to more accurately describe the richness and diversity of a specific microbiome, essentially superseding Sanger 16S rRNA gene sequencing for bacterial community analysis.

Bacterial 16S rRNA gene sequencing has been used to elucidate the composition of the oral microbiome of some other animal species. Kennedy et al. (2016) identified 1308 operative taxonomic units in the oral microbiome of horses. The genera Gemella and Actinobacillus were the most abundant in samples of periodontally healthy animals, whereas in the group of animals with periodontitis the genera Prevotella and Veillonella prevailed.

In periodontally healthy dogs, Dewhirst et al. (2012) identified 353 taxa, which were placed in 14 bacterial phyla, 23 classes, 37 orders, 66 families, and 148 genera. Eighty percent of identified taxa were unnamed. Holcombe et al. (2014) evaluated the colonisation of the supragingival surface of canine teeth and identified a total of 134 specieslevel operative taxonomic units that were distributed among the phyla Proteobacteria, Bacteroidetes, Firmicutes, Actinobacteria and Fusobacteria. 


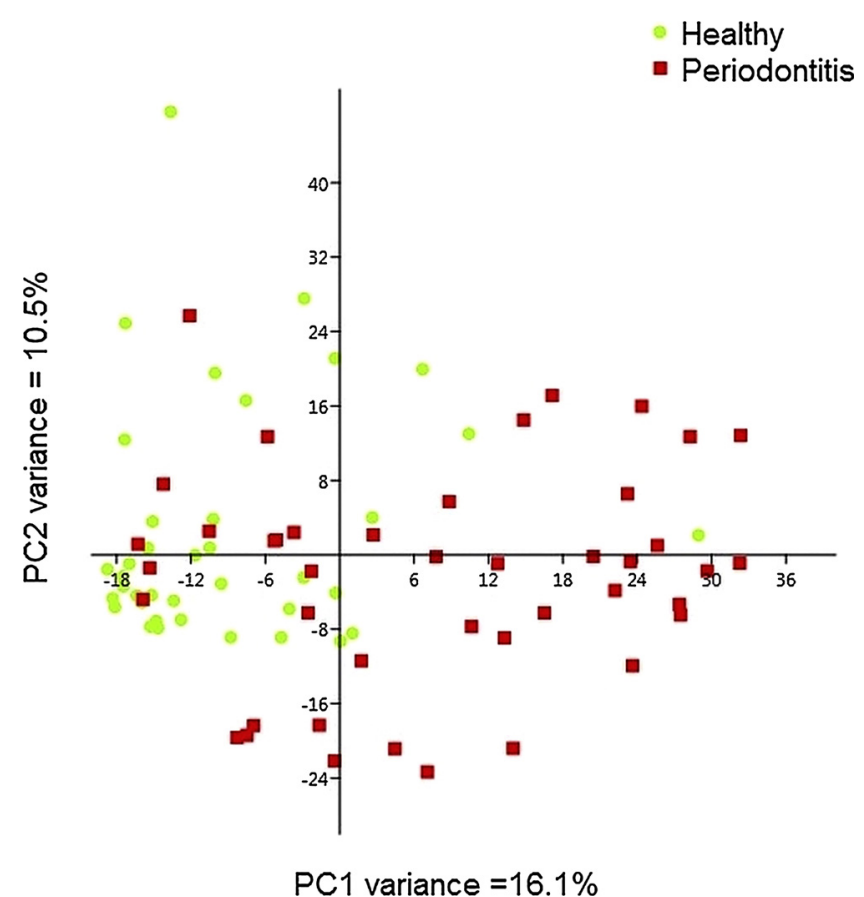

Fig. 2. Two-dimensional ordination of bovine microbial profiles in oral health and periodontitis by principal component analysis (PCA). Identified OTUs were randomly subsampled to $50 \%$ and $\log _{2}$-transformed prior to the PCA.

In cats, Sturgeon et al. (2014) identified 10177 OTUs in the oral microbiome of healthy animals, representing 18 phyla, of which the most prevalent were Proteobacteria $(75.2 \%)$, Bacteroidetes $(9.3 \%)$, Firmicutes (6.7\%), Spirochaetes (1.8\%), Fusobacteria (1.3\%) and Actinobacteria $(0.6 \%)$. The most prevalent genera were Moraxella (10.9\%), Thermomonas (6.9\%), Neisseria (4.9\%) and Pasteurella (4.3\%).

In the present study, the taxa Gastranaerophilales, Planifilum, Burkholderia and Arcobacter were the most prevalent in healthy animals, while Elusimicrobia, Synergistes and Propionivibrio were most frequently observed in the oral microbiota of cattle with periodontitis. However, little is known regarding these microorganisms. Fusobacteria, Wolinella, Porphyromonas, Prevotella and Treponema were also found at high prevalence in bovine periodontitis lesions.

The Fusobacteria phylum, which contains bacteria of the genus Fusobacterium, has been recognised as part of the subgingival microbiota for more than 100 years. In cattle, Fusobacterium nucleatum was detected in culture of periodontitis lesions (Blobel et al., 1987; Botteon et al., 1993). Fusobacterium naviforme, Fusobacterium necrophorum and $F$. nucleatum have been identified in sheep with 'broken mouth' periodontitis (McCourtie et al., 1989) and F. necrophorum has been identified in goats with periodontitis (Suzuki et al., 2006).

The Fusobacterium genus is one of the main constituents of the normal oral microbiota of cats (Love et al., 1990) and several species of the genus, such as Fusobacterium alocis (Hardham et al., 2005), Fusobacterium canifelinum (Conrads et al., 2004; Dahlén et al., 2012) and F. nucleatum (Nishiyama et al., 2007), have been detected in dogs with and without periodontitis.

Black-pigmented bacteria of the genera Porphyromonas and Prevotella are recognised pathogens in human and animal periodontitis. Different species of both genera have been identified in dogs (Hardham et al., 2005; Nishiyama et al., 2007; Riggio et al., 2011), cats (Booij-Vrieling et al., 2010) and sheep with periodontitis (McCourtie et al., 1989, Duncan et al., 2003; Riggio et al., 2013; Borsanelli et al., 2017).

In cattle, these two genera appear to play an important role in the lesions of animals with periodontitis (Blobel et al., 1987; Botteon et al., 1993; Dutra et al., 1986, 2000). When evaluating the presence of Prevotella and Porphyromonas species in the bovine microbiota with and without periodontitis, Borsanelli et al. (2015b) found that the occurrence of Porphyromonas asaccharolytica, Porphyromonas endodontalis, Prevotella buccae, Prevotella intermedia, Prevotella melaninogenica and Prevotella oralis was associated with bovine periodontitis.

There are a variety of quantitative and qualitative studies that evaluated Treponema species involved in human periodontitis or healthy sites (Sato and Kuramitsu, 2000; Asai et al., 2002), as well as in dogs with periodontitis (Riviere et al., 1996; Nordhoff et al., 2008). Several species of the genus Treponema were identified in periodontal lesions of sheep (Borsanelli et al., 2016b), and in cattle Treponema amylovorum, Treponema maltophilum and Treponema denticola were detected in the microbiota of animals with periodontitis (Borsanelli et al., 2015a). This genus was also found at high levels in horses with periodontitis (Kennedy et al., 2016).

No previous study has characterised the bovine oral microbiome in as much detail as presented in the current study. Samples from bovine oral health and periodontitis had different microbial profiles, but the diversity of the bacteria found in health and periodontitis were similar; bacteria commonly recognised as periodontal pathogens showed an increased abundance in disease. In this context, the main components of bacterial homeostasis in the biofilm of healthy sites and of dysbiosis in periodontal lesions provide unprecedented indicators for the evolution of knowledge about bovine periodontitis.

\section{Conflicts of interest}

The authors have no conflicts of interest.
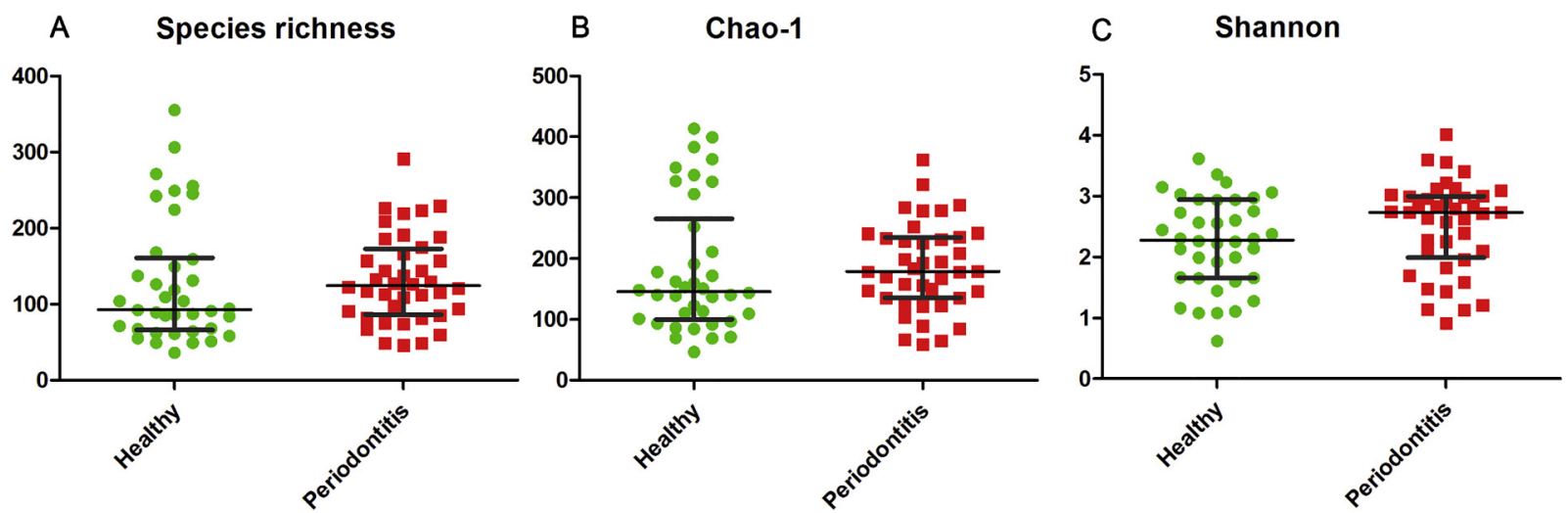

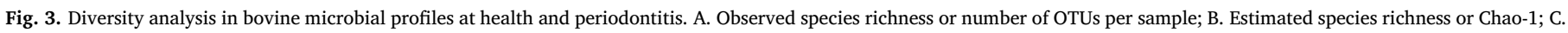
Shannon diversity index. 


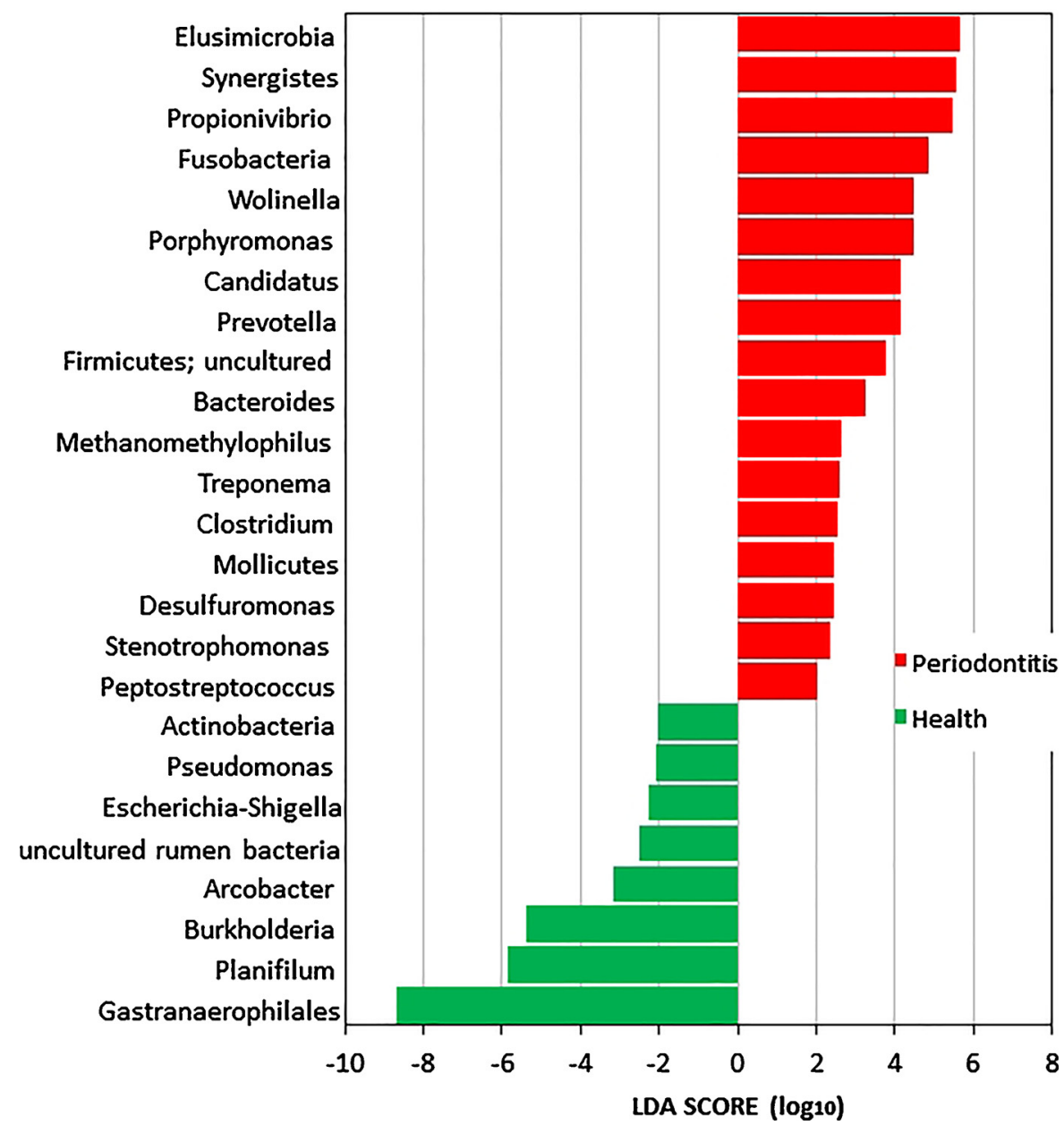

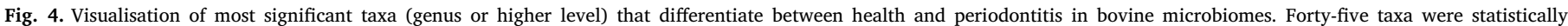
significantly different between the two groups. Only taxa with an LDA score of two or above are shown. Taxa are ranked by the effect size in LEfSe.

\section{Acknowledgement}

This work was supported by a scholarship from the São Paulo Research Foundation (FAPESP), (Process FAPESP nº 2015/06917-9).

\section{References}

Aitchison, G.U., Spence, J.A., 1984. Dental disease in hill sheep: an abattoir survey. J. Comp. Pathol. 94, 285-300.

Asai, Y., Jinno, T., Igarashi, H., Ogawa, T., 2002. Detection and quantification of oral treponemes in subgingival plaque by real-time PCR. J. Clin. Microbiol. 40, 3334-3340.

Blobel, H., Döbereiner, J., Rosa, I.V., Lima, F.G.F., Dutra, I.S., 1987. Bacterial investigations of a periodontal disease, Cara inchada in Brazilian cattle. Tierärztliche Umschau 42, 152-154.

Booij-Vrieling, H.E., van der Reijden, W.A., Houwers, D.J., de Wit, W.E.A.J., Bosch-Tijhof, C.J., Penning, L.C., van Winkelhoff, A.J., Hazewinkel, H.A., 2010. Comparison of periodontal pathogens between cats and their owners. Vet. Microbiol. 144, 147-152.

Borsanelli, A.C., Gaetti-Jardim Jr, E., Döbereiner, J., Dutra, I.S., 2015a. Treponema denticola in microflora of bovine periodontitis. Pesq. Vet. Bras. 35, 237-240.

Borsanelli, A.C., Gaetti-Jardim Jr, E., Schweitzer, C.M., Döbereiner, J., Dutra, I.S., 2015b. Presence of Porphyromonas and Prevotella species in the oral microflora of cattle with periodontitis. Pesq. Vet. Bras. 35, 829-834.

Borsanelli, A.C., Viora, L., Lappin, D.F., Bennett, D., King, G., Dutra, I.S., Riggio, M.P., 2016a. Periodontal lesions in slaughtered cattle in the west of Scotand. Vet. Rec. 179, 652.

Borsanelli, A.C., Ramos, T.N.M., Gaetti-Jardim Jr., E., Schweitzer, C.M., Dutra, I.S., 2016b. Treponema species in the subgingival microflora of ovine periodontitis. Vet Rec. 180, 150.

Borsanelli, A.C., Gaetti-Jardim Jr., E., Schweitzer, C.M., Viora, L., Busin, V., Riggio, M.P., Dutra, I.S., 2017. Black-pigmented anaerobic bacteria associated with ovine periodontitis. Vet. Microbiol. 203, 271-274.

Botteon, R.M., Dutra, I.S., Döbereiner, J., Blobel, H., 1993. Characterization of anaerobic bacteria isolated from peridental lesions of bovine "swollen face". Pesq. Vet. Bras. 13,
$51-55$.

Caporaso, J.G., Kuczynski, J., Stombaugh, J., Bittinger, K., Bushman, F.D., Costello, E.K. Fierer, N., Peña, A.G., Goodrich, J.K., Gordon, J.I., Huttley, G.A., Kelley, S.T., Knights, D., Koenig, J.E., Ley, R.E., Lozupone, C.A., McDonald, D., Muegge, B.D., Pirrung, M., Reeder, J., Sevinsky, J.R., Turnbaugh, P.J., Walters, W.A., Widmann, J., Yatsunenko, T., Zaneveld, J., Knight, R., 2010. QIIME allows analysis of highthroughput community sequencing data. Nat. Methods 7, 335-336.

Chen, T., Yu, W.H., Izard, J., Baranova, O.V., Lakshmanan, A., Dewhirst, F.E., 2010. The Human Oral Microbiome Database: A Web Accessible Resource for Investigating Oral Microbe Taxonomic and Genomic Information. Database (Oxford) 2010, baq013. http://dx.doi.org/10.1093/database/baq013.

Cole, J.R., Wang, Q., Cardenas, E., Fish, J., Chai, B., Farris, R.J., Kulam-Syed-Mohideen, A.S., McGarrell, D.M., Marsh, T., Garrity, G.M., Tiedje, J.M., 2009. The ribosomal database project: improved alignments and new tools for rRNA analysis. Nucleic Acids Res. 37, D141-D145.

Conrads, G., Citron, D.M., Mutters, R., Jang, S., Goldstein, E.J.C., 2004. Fusobacterium canifelinum sp. nov., from the oral cavity of cats and dogs. Syst. Appl. Microbiol. 27, 407-413.

Dahlén, G., Charalampakis, G., Abrahamsson, I., Bengtsson, L., Falsen, E., 2012 Predominant bacterial species in subgingival plaque in dogs. J. Periodont. Res. 47, 354-364.

Dewhirst, F.E., Klein, E.A., Thompson, E.C., Blanton, J.M., Chen, T., Milella, L., Buckley, C.M.F., Davis, J.A., Bennett, M.L., Marshall-Jones, Z.V., 2012. The canine oral microbiome. PLoS One 7 (4), e36067.

Döbereiner, J., Dutra, I.S., Rosa, I.V., Blobel, H., 2000. "Cara inchada" of cattle, an infectious, apparently soil antibiotics-dependant periodontitis in Brazil. Pesq. Vet. Bras. 20, 47-64.

Duncan, W.J., Persson, G.R., Sims, T.J., Braham, P., Pack, A.R.C., Page, R.C., 2003. Ovine periodontitis as a potential model for periodontal studies. J. Clin. Periodontol. 30, 63-72.

Dutra, I.S., Botteon, R.C.M., Döbereiner, J., 2000. Modification of the microflora associated with the periodontal lesions of "cara inchada" in calves transferred to a disease-free area. Pesq. Vet. Bras. 20, 71-74.

Dutra, I.S., Kanoe, M., Blobel, H., 1986. Enzymatic and endotoxic activities of bacteria isolated from peridental lesions of the "swollen face" of cattle. Pesq. Vet. Bras. 6, 59-63. 
Edgar, R.C., Flyvbjerg, H., 2015. Error filtering, pair assembly and error correction for next-generation sequencing reads. Bioinformatics 31, 3476-3482.

Fadden, A.N., Poulsen, K.P., Vanegas, J., Mecham, J., Bildfell, R., Stieger-Vanegas, S.M., 2015. Dental pathology in conventionally fed and pasture managed dairy cattle. Vet. Rec. 178, 19.

Hajishengallis, G., Lamont, R.J., 2012. Beyond the red complex and into more complexity: the polymicrobial synergy and dysbiosis (PSD) model of periodontal disease etiology. Mol. Oral Microbiol. 27, 409-419.

Hammer, Ø., Harper, D.A.T., Ryan, P.D., 2001. PAST: paleontological statistics software package for education and data analysis. Palaeontol. Electronica 4, 9.

Hardham, J., Dreier, K., Wong, J., Sfintescu, C., Evans, R.T., 2005. Pigmented-anaerobic bacteria associated with canine periodontitis. Vet. Microbiol. 106, 119-128.

Holcombe, L.J., Patel, N., Colyer, A., Deusch, O., O'Flynn, C., Harris, S., 2014. Early canine plaque biofilms: characterization of key bacterial interactions involved in initial colonization of enamel. PLoS One 9 (12), e113744.

Ingham, B., 2001. Abbatoir survey of dental defects in cull cows. Vet. Rec. 148, 739-742.

Kennedy, R., Lappin, D.F., Dixon, P.M., Buijs, M.J., Zaura, E., Crielaard, W., O’Donnell, L., Bennett, D., Brandt, B.W., Riggio, M.P., 2016. The microbiome associated with equine periodontitis and oral health. Vet. Res. 47, 49.

Love, D.N., Vekselstein, R., Collings, S., 1990. The obligate and facultatively anaerobic bacterial flora of the normal feline gingival margin. Vet. Microbiol. 22, 267-275.

McCourtie, J., Poxton, I.R., Spence, J.A., Aitchison, G.U., 1989. Preliminary study of the anaerobic bacteria isolated from subgingival plaque from sheep. Vet. Microbiol. 21, 139-146.

Nishiyama, S.A.B., Senhorinho, G.N.A., Gioso, M.A., Avila-Campos, M.J., 2007. Detection of putative periodontal pathogens in subgingival specimens of dogs. Braz. J. Microbiol. 38, 23-28.

Nordhoff, M., Rühe, B., Kellermeier, C., Moter, A., Schmitz, R., Brunnberg, L., Wieler,
L.H., 2008. Association of Treponema spp. with canine periodontitis. Vet. Microbiol. 27, 334-342.

Page, R.C., Schroeder, H.E., 1976. Pathogenesis of inflammatory periodontal disease: a summary of current work. Lab. Invest. 33, 235-249.

Quast, C., Pruesse, E., Yilmaz, P., Gerken, J., Schweer, T., Yarza, P., Peplies, J., Glöckner, F.O., 2013. The SILVA ribosomal RNA gene database project: improved data processing and web-based tools. Nucleic Acids Res. 41 (D1), D590-D596.

Riggio, M.P., Lennon, A., Taylor, D.J., Bennett, D., 2011. Molecular identification of bacteria associated with canine periodontal disease. Vet. Microbiol. 150, 394-400.

Riggio, M.P., Jonsson, N., Bennett, D., 2013. Culture-independent identification of bacteria associated with ovine 'broken mouth' periodontitis. Vet. Microbiol. 166, 664-669.

Riviere, G.R., Thompson, A.J., Brannan, R.D., McCoy, D.E., Simonson, L.G., 1996. Detection of pathogen-related oral spirochetes, Treponema denticola, and Treponema socranskii in dental plaque from dogs. J. Vet. Dent. 13, 135-138.

Sato, T., Kuramitsu, H.K., 2000. Polymerase chain reaction for the detection of flaA-1 genes of oral spirochetes in human advanced periodontal pockets. Arch. Oral Biol. 45, 921-925.

Segata, N., Izard, J., Waldron, L., Gevers, D., Miropolsky, L., Garrett, W.S., Huttenhower, C., 2011. Metagenomic biomarker discovery and explanation. Genome Biol. 12, R60.

Socransky, S.S., Gibbons, R.J., Dale, A.C., Bortnick, L., Rosenthal, E., Macdonald, J.B., 1963. The microbiota of the gingival crevice of man - I: total microscopic and viable counts and counts of specific organisms. Arch. Oral Biol. 8, 275-280.

Sturgeon, A., Pinder, S.L., Costa, M.C., Weese, J.S., 2014. Characterization of the oral microbiota of healthy cats using next-generation sequencing. Vet. J. 201, 223-229. Suzuki, S., Mitani, A., Koyasu, K., Oda, S.I., Yoshinari, N., Fukuda, M., Hanamura, H., Nakagaki, H., Noguchi, T., 2006. A model of spontaneous periodontitis in the miniature goat. J. Periodontol. 77, 847-855. 